\title{
EPR spectra of deuterated methyl radicals trapped in low temperature matrices
}

\author{
Yu.A. Dmitriev \\ A.F. Ioffe Physico-Technical Institute, 26 Politekhnicheskaya Str., St. Petersburg 194021, Russia \\ E-mail:dmitriev.mares@pop.ioffe.rssi.ru
}

Received August 2, 2004, revised November 2, 2004

\begin{abstract}
EPR spectra of $\mathrm{CHD}_{2}$, and $\mathrm{CD}_{3}$ radicals have been investigated in low-temperature matrices of $\mathrm{H}_{2}, \mathrm{D}_{2}$, and $\mathrm{Ne}$ at temperatures 1.6-4.2 K. A method of condensation from the gas phase on a cold substrate has been used. With decreasing sample temperature, a transformation of the shape of the $\mathrm{CD}_{3}$ spectrum in $\mathrm{H}_{2}, \mathrm{D}_{2}$, and $\mathrm{Ne}$ matrices and $\mathrm{CHD}_{2}$ spectrum in $\mathrm{H}_{2}$ and $\mathrm{Ne}$ was observed. This transformation was reversible in the above temperature range. The temperature effects are explained as reflecting a change in the populations of the lowest rotational states of the radicals. Based on the present data and known results for deuterated methyl radicals in Ar obtained in photolytic experiments, we compare the temperature behavior of the EPR spectra for the radicals trapped in various matrices. As a result an existence of a hindering barrier for the radical rotation is suggested.
\end{abstract}

PACS: 32.30.-r, 76.30.-v

\section{Introduction}

Methyl radical $\left(\mathrm{CH}_{3}\right)$ trapped in low-temperature matrices has been extensively studied by EPR [1-8]. It was shown that at low temperatures near $4.2 \mathrm{~K}$, the EPR spectrum of the radical consists of four lines with equal intensity $1: 1: 1: 1$, reaching the high-temperature binomial intensity distribution 1:3:3:1 with increasing temperature. In explaining the EPR spectrum shape and its transformation, two theoretical approaches are used: one treating the $\mathrm{CH}_{3}$ radical as a restricted rotor, and another - as a free rotor. In contrast to $\mathrm{CH}_{3}$, a few studies have been devoted to deuterated methyl radicals $\left(\mathrm{CD}_{3}, \mathrm{CH}_{2} \mathrm{D}\right.$ and $\left.\mathrm{CHD}_{2}\right)$. A spectrum of seven components with «non-binomial» distribution has been predicted for the $\mathrm{CD}_{3}$ radical [9] at low enough temperatures. The septet has been actually registered in the $\mathrm{CD}_{4}$ matrix [10] at $4.2 \mathrm{~K}$ and solid $\mathrm{Ar}$ at $13 \mathrm{~K}$ [4]. Though these experimental results are consistent with the above theoretical scheme, another observation has been published [5] for $\mathrm{CD}_{3}$ in $\mathrm{Ar}$ at $4.2 \mathrm{~K}$ showing a strong singlet superimposed on a weak septet. The authors explained their results with a new model of a three-dimensional, free quantum rotor with no hindering barrier present. They pointed out that the electronic state has to be included in the application of the Pauli principle in order to obtain correct overall exchange symmetry for bosons.

In Ref. 11, results were presented on deuterated methyl radicals trapped from the gas phase in solid $\mathrm{H}_{2}$. The temperatures at which the $\mathrm{CHD}_{2}$ and $\mathrm{CD}_{3}$ spectrum changed to the low-temperature shape turned out to be surprisingly low in $\mathrm{H}_{2}$ matrix as compared to solid Ar [5]. The present study is aimed at obtaining new information about deuterated methyl radicals in $\mathrm{H}_{2}$ and studying these radicals in solid $\mathrm{D}_{2}$ and $\mathrm{Ne}$ in order to clarify whether the effect found in [5] is common to other matrices and to correlate new results with each other, which would help to obtain new data about the matrix effect on the radical rotation.

\section{Results and discussion}

The experimental technique and a schematic of the setup have been presented elsewhere (e. g., [12])

In our experiment, molecular deuterium, $\mathrm{D}_{2}$, mixed with $4 \mathrm{~mol} \%$ methane, $\mathrm{CH}_{4}$, was prepared in a glass vessel and passed through a discharge zone onto a low temperature substrate. Simultaneously, the $\mathrm{D}_{2}$ was fed onto the same substrate through a separate inlet tube to avoid the gas discharge. The latter flow was much larger than the discharge flow. We estimated an 
admixture of $\mathrm{CH}_{4}$ in $\mathrm{D}_{2}$ matrix to be about $0.5-1 \%$. A pulsed discharge has been employed with the off-duty factor of 7 . The substrate temperature during the deposition was $4.2 \mathrm{~K}$. Figure 1 shows the EPR spectrum of a sample of solid $\mathrm{D}_{2}$ with trapped radicals. For reference, a scheme of EPR transitions for $\mathrm{CH}_{2} \mathrm{D}$, $\mathrm{CHD}_{2}$, and $\mathrm{CD}_{3}$ radicals at high (all rotation levels are populated) and low (only the lowest rotational level is populated) temperatures is shown in Fig. 2, according to [5].

The experimental spectrum reveals seven strong lines of the $\mathrm{CD}_{3}$ radical and several weak lines for $\mathrm{CH}_{3}, \mathrm{CH}_{2} \mathrm{D}$, and $\mathrm{CHD}_{2}$ radicals. Superimposed is a record of the high-field lines with a higher gain. Due to the rather large broadening of the lines (the peak-to-peak linewidth $\Delta H=1.15(5) \mathrm{G}$ ) only some components of the $\mathrm{CH}_{3}, \mathrm{CH}_{2} \mathrm{D}$, and $\mathrm{CHD}_{2}$ spectra can be resolved. The $\mathrm{CH}_{3}$ spectrum is supposedly composed of four lines of equal intensity, like the $\mathrm{CH}_{3}$ spectrum in the $\mathrm{H}_{2}$ matrix studied earlier [6]. The $\mathrm{CH}_{2} \mathrm{D}$ spectrum is a triple triplet due to the hyperfine (hf) splittings of the two hydrogen nuclei, the major triplet, and one deuterium, the minor triplets. At high enough temperatures, the $\mathrm{CHD}_{2}$ spectrum is a double quintet.

We have found that two adjacent $\mathrm{CD}_{3}$ lines are spaced with $\delta H=3.56(4) \mathrm{G}$. It was observed in Ref. 5 that at temperatures above $10 \mathrm{~K}$ the intensity distribution for $\mathrm{CD}_{3}$ in $\mathrm{Ar}$ is practically «binomial» one, 1:3:6:7:6:3:1, originating not only from the population of $J=0$ but also of higher rotational levels. As the temperature was lowered to $4.1 \mathrm{~K}$ the central line

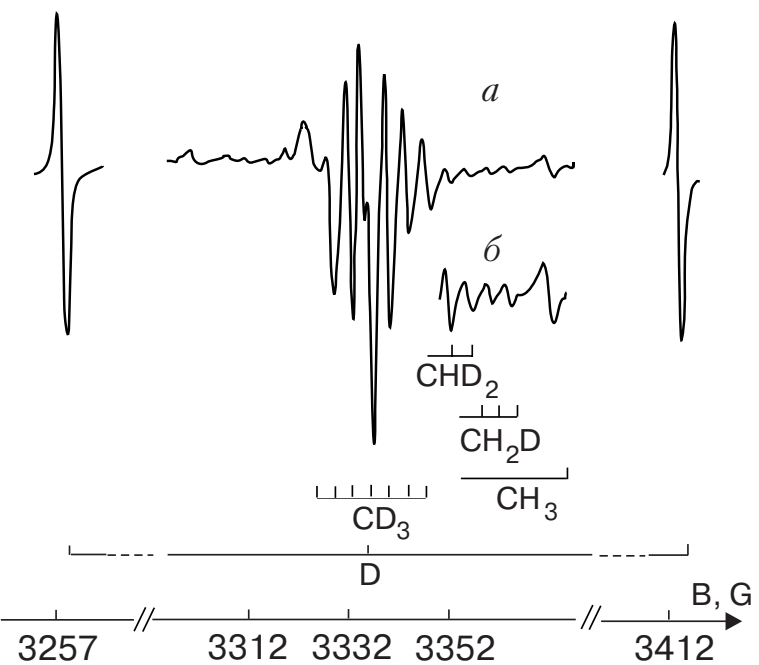

Fig. 1. The EPR spectrum of a solid $\mathrm{D}_{2}$ sample at $4.2 \mathrm{~K}$ with trapped methyl radicals. Also shown are the D-atom transitions $(a)$. The high-field part of the spectrum with higher gain $(b)$. The substrate temperature during deposition $T_{\text {sub }}=4.2 \mathrm{~K}$. Deposition duration, $\tau_{\text {dep }} \approx 75 \mathrm{~min}$. EPR resonance frequency $f_{\text {res }}=9348.22 \mathrm{MHz}$. $a$

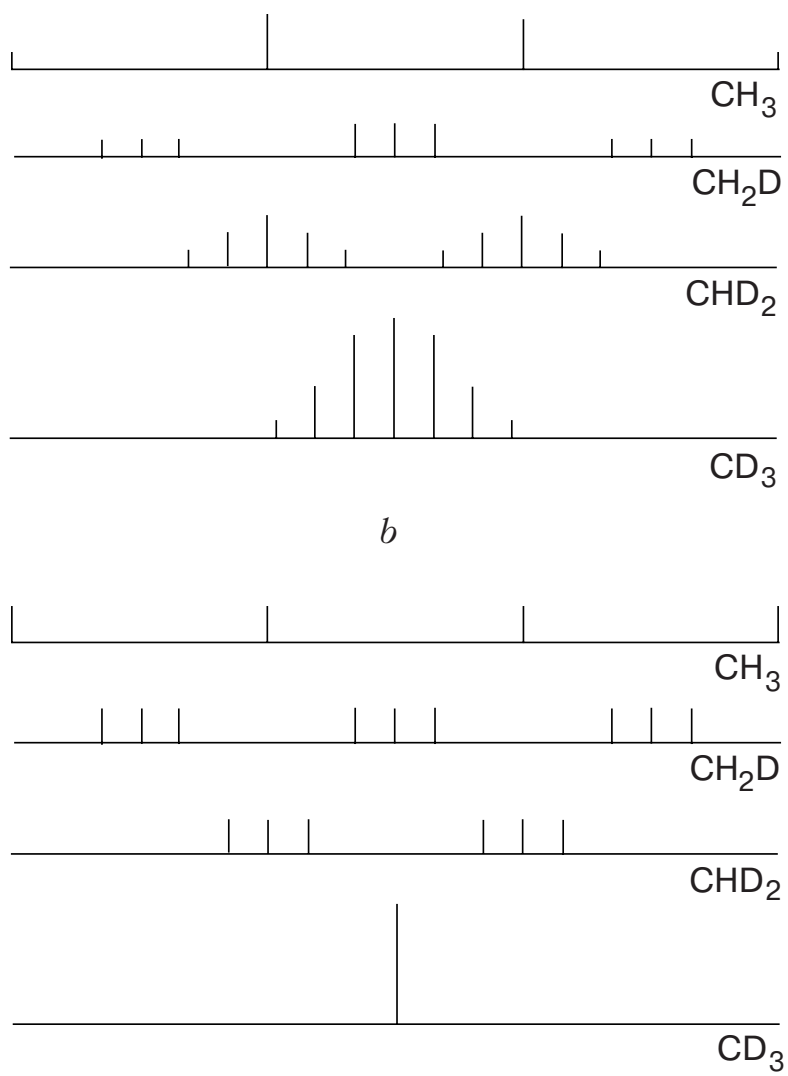

Fig.2. The scheme of $\mathrm{CH}_{3}, \mathrm{CH}_{2} \mathrm{D}, \mathrm{CHD}_{2}$, and $\mathrm{CD}_{3}$ transitions at $(a)$ high and $(b)$ low temperatures.

increased while the other six lines decreased rapidly. As a result, the intensity of the central peak relative to the adjacent one reached 15 . It was shown that the spectrum corresponding to the $J=0$ rotation level is a singlet. Turning to our study, it is hard to estimate the relative intensity ratio because the lines are mostly superimposed. However, the amplitude ratio of two «pure» $\mathrm{CD}_{3}$ lines, that is third to second (counting from left), equals 2, which is expected for the binomial distribution. The line superimposed on the central $\mathrm{CD}_{3}$ peak in Fig. 1 is a central transition of the D-atom triplet. We have also recorded other two D-atom lines which appeared alone, i.e., not as a mixture with any transitions. These lines (shown in Fig. 1) were used to extract the «pure» $\mathrm{CD}_{3}$ central line by subtracting D-atom transition, Fig. 3.

As a result, the amplitude ratio of the central line to its nearest neighbor was estimated to be 1.32 which is a bit larger then the binomial 7:6. Thus the spectrum of $\mathrm{CD}_{3}$ in $\mathrm{D}_{2}$ at $4.2 \mathrm{~K}$ is a high-temperature nearly «binomial» spectrum with possibly a small admixture of the low-temperature singlet. The above mentioned $\mathrm{CH}_{3}$ spectrum of four equal lines corresponds to $J=0$, thus being a low-temperature one. Such a difference between the appearances of the $\mathrm{CH}_{3}$ and $\mathrm{CD}_{3}$ spectra is not surprising because the energy 


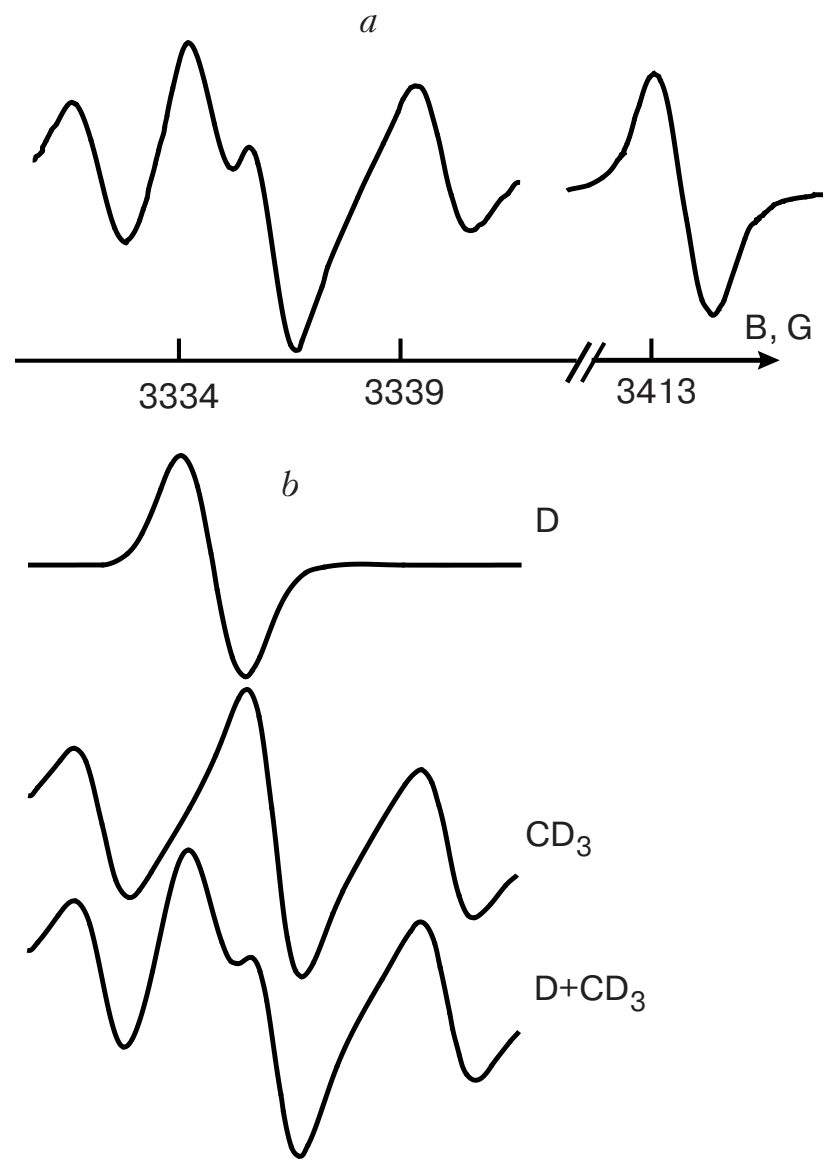

Fig. 3. Central part of the EPR spectra of the $\mathrm{CD}_{3}$ radical and $\mathrm{D}$-atom lines in $\mathrm{D}_{2}$ matrix. The substrate temperature during deposition, $T_{\text {sub }}=4.2 \mathrm{~K}$. The substrate temperature during registration, $T_{\text {reg }}=2.85 \mathrm{~K}(a)$. The simulated central part of the $\mathrm{CD}_{3}$ spectrum with the central D-atom transition superimposed (b).

gap between the $J=0$ and $J=1$ rotational states for the free $\mathrm{CH}_{3}$ is twice as large as that for the free $\mathrm{CD}_{3}$. Therefore, the $J=1$ state of the $\mathrm{CH}_{3}$ radical is not populated at low temperatures close to $4 \mathrm{~K}$. We have

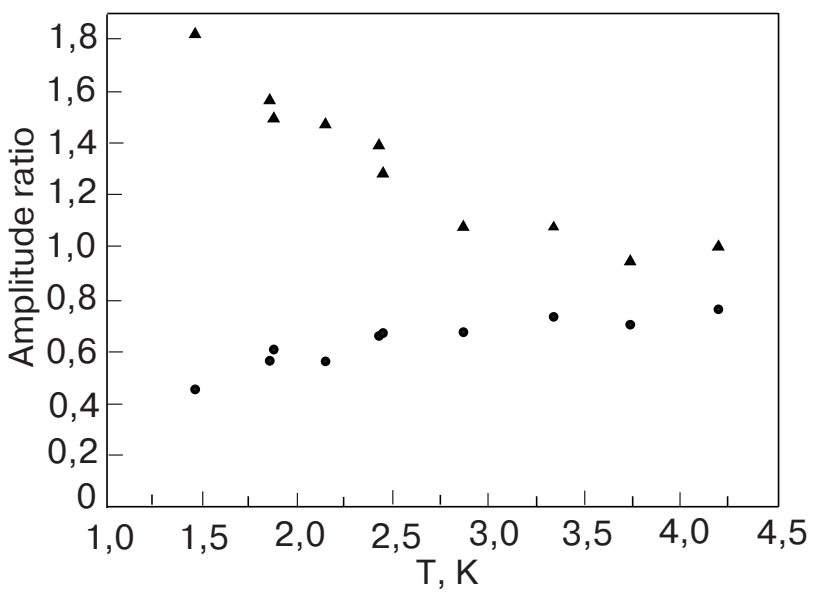

Fig. 4. Amplitudes of the $\mathrm{CD}_{3}$ central line $(\boldsymbol{\Delta})$ and its nearest high-field neighbor ( $)$ versus temperature. The $\mathrm{D}$-atom transition is used as a reference signal.

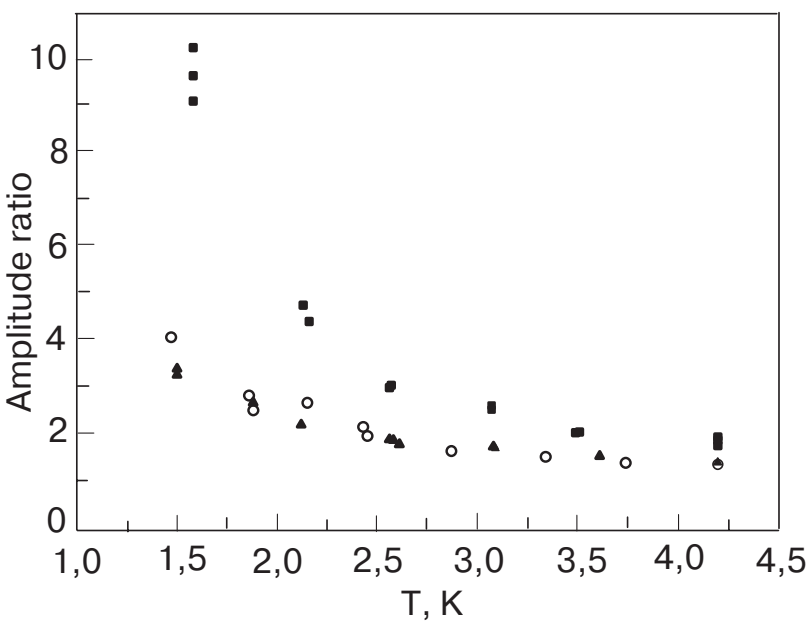

Fig. 5. The temperature dependence of the relative intensity of the $\mathrm{CD}_{3}$ central component $\left(m_{F}=0\right)$ to the neighbor transitions $\left(m_{F}= \pm 1\right)$ for the $\mathrm{CD}_{3}$ radical trapped in low-temperature solids: $\mathrm{H}_{2}(\boldsymbol{\bullet}), \mathrm{D}_{2}(\mathrm{O})$, and $\mathrm{Ne}(\boldsymbol{\Delta})$.

found a change in the appearance of the $\mathrm{CD}_{3}$ spectrum with varying temperature. The high-field D-atom transition was used as a reference signal. Figure 4 shows a plot of the amplitudes of the central $\mathrm{CD}_{3}$ line and its nearest high-field neighbor versus temperature. One can readily see that the central peak with $m_{F}=0$ increases with decreasing temperature, while the amplitude of the other line decreases. Plotting these data, we took special care to avoid line saturation due to microwave power. Such a difference in the temperature behavior between the lines is proof that the central peak is actually a superposition of transitions due to different rotational states: $J=0$ and $J=1$. The population of the lowest $J=0$ level increases with decreasing temperature, while the population of higher rotational levels decreases. This temperature behavior also manifests itself in Fig. 5, which shows the amplitude ratio of the central line to its nearest neighbor.

An investigation has been undertaken into correlation between the temperature change of the EPR spectrum shape and the saturation behavior for the $\mathrm{CHD}_{2}$ and $\mathrm{CD}_{3}$ in the molecular hydrogen matrix. Figure 6 shows the saturation curves, that is, the intensities of the central and adjacent lines versus microwave power. The high-temperature spectrum experienced no saturation in the power range used at both $4.2 \mathrm{~K}$ and $1.6 \mathrm{~K}$, whereas the central peak starts to saturate at $4.2 \mathrm{~K}$, reaching a pronounced saturation at $1.6 \mathrm{~K}$. Such a difference in the saturation behavior between the lines is further proof that the central peak is actually a superposition of transitions due to different states.

The solid Ne sample with trapped methyl radicals was prepared by co-condensation on a substrate at 

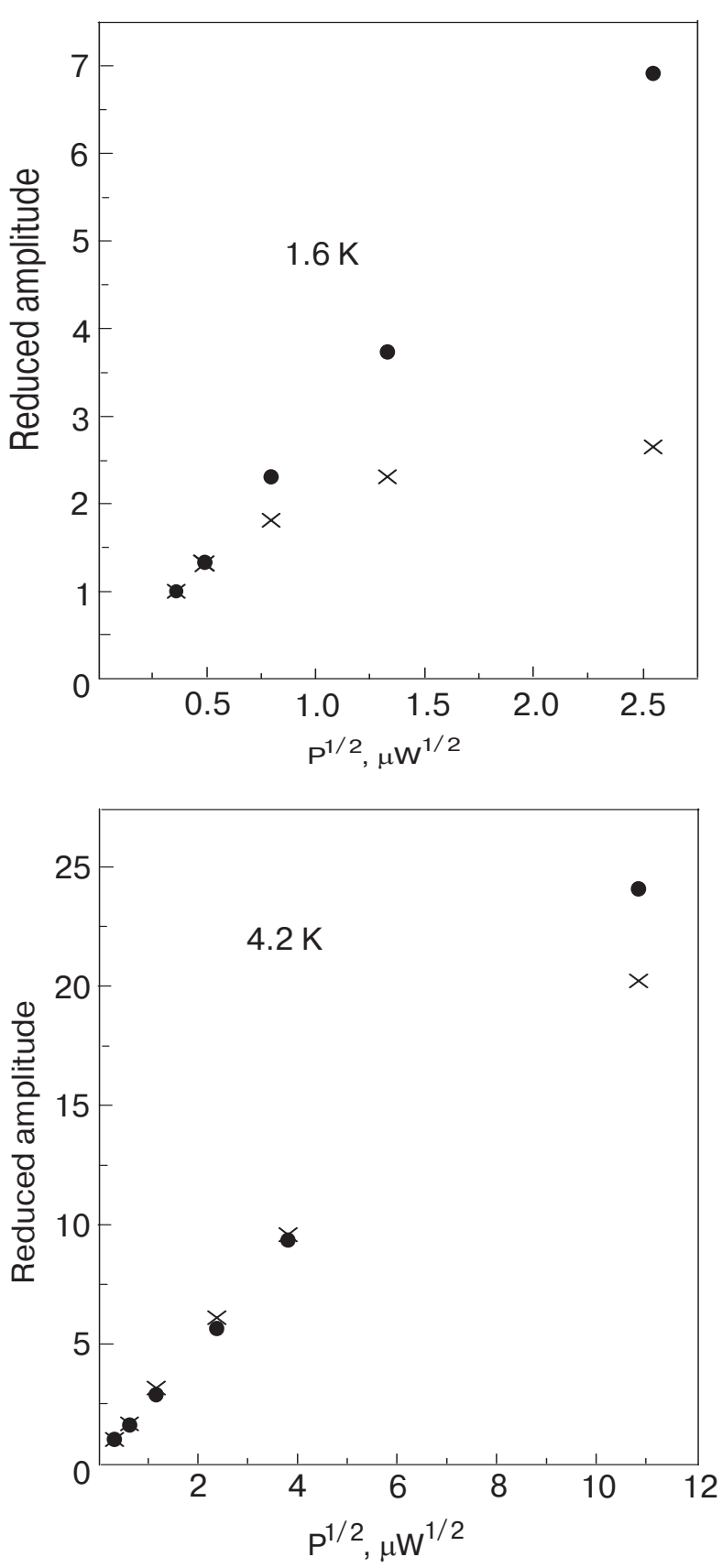

Fig. 6. The amplitudes of the $\mathrm{CD}_{3}$ central, $m_{F}=0(\times)$ and neighbor, $m_{F}= \pm 1(\bullet)$ transitions versus microwave power for the radical trapped in solid $\mathrm{H}_{2}$. For a convenience of the comparison, the amplitudes of the components are set equal at the lowest microwave power.

$4.2 \mathrm{~K}$ of two flows: a Ne flow with $1 / 3$ impurity of $\mathrm{D}_{2}$ mixed with $5 \mathrm{~mol} \%$ methane, and a pure Ne flow through the matrix channel. The impurity concentration in the Ne matrix was estimated to be $1-1.5 \%$. A pulsed discharge was employed. The substrate temperature during deposition was $4.2 \mathrm{~K}$. As a result, we obtained a well-resolved spectrum (Fig. 7) of rather narrow lines of the radicals, with a linewidth of about $0.2-0.25 \mathrm{G}$. The relative intensity ratio for the $\mathrm{CD}_{3}$ radical, $1: 2.9: 5.4: 7.5: 4.8: 2.8: 1$, is close to the

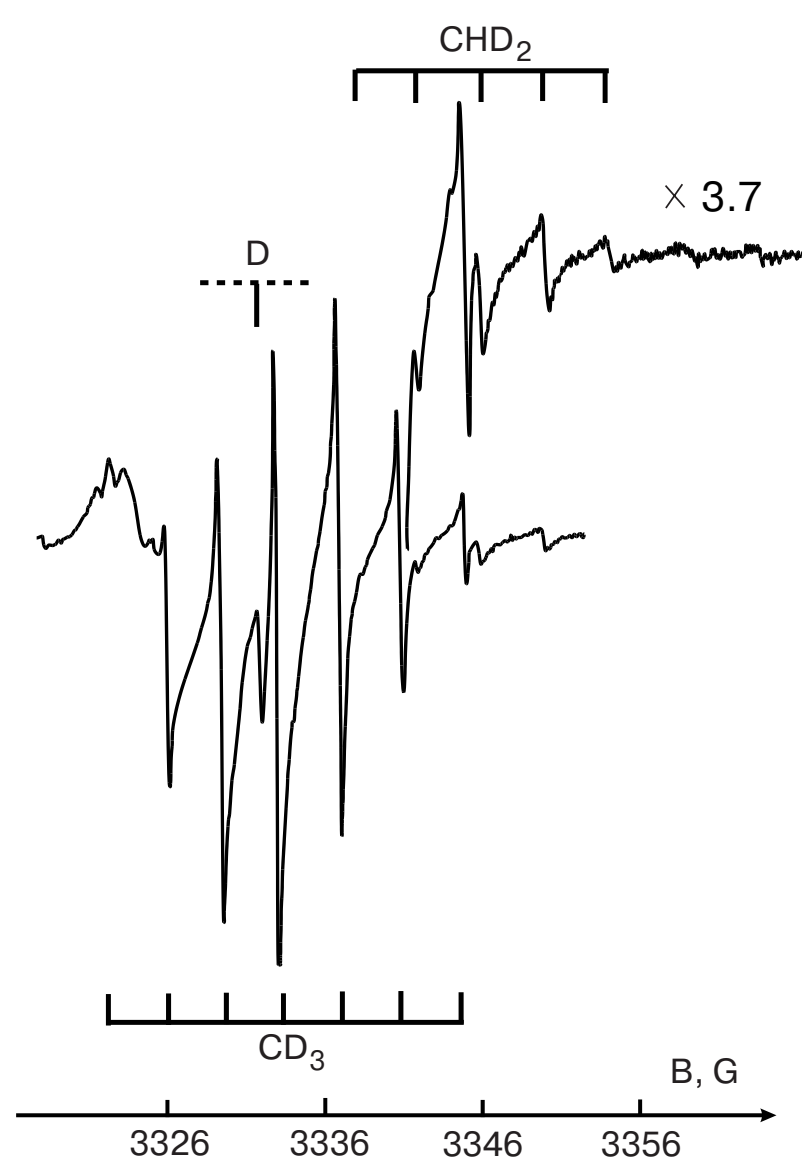

Fig. 7. The EPR spectrum of a solid Ne sample at $4.2 \mathrm{~K}$ with trapped methyl radicals. Also shown is the high-field part of the spectrum with higher gain. The substrate temperature during deposition $T_{\text {sub }}=4.2 \mathrm{~K}, \tau_{\text {dep }} \approx 1 \mathrm{~h}, f_{\text {res }}=$ $=9353.43 \mathrm{MHz}$.

«binomial» intensity distribution 1:3:6:7:6:3:1. In these experiments, we observed an increase of the central line amplitude relative to intensities of other six lines. The ratio of the central line amplitude to the amplitude of its nearest high-temperature neighbor is presented in Fig. 5. Figure 8 shows the central part of the $\mathrm{CD}_{3}$ spectrum in Ne taken at several temperatures. One can readily see that the central peak at $m_{F}=0$ increases rapidly with decreasing temperature in relation to the neighboring transitions.

The investigation with the Ar matrix is still in progress, because in our attempts to study $\mathrm{CD}_{3}$ trapped in a solid Ar sample we encounter the problem of a very strong central line of the D-atom triplet superimposed on a weaker $\mathrm{CD}_{3}$ spectrum. In this situation, any measurements would be of insufficient reliability. We did not face this difficulty with the $\mathrm{Ne}$ and $\mathrm{D}_{2}$ matrices because $\mathrm{D}$ atoms are usually trapped in $\mathrm{Ne}$ and $\mathrm{D}_{2}$ in much lower concentrations than in Ar. With the $\mathrm{H}_{2}$ matrix, the $\mathrm{D}$ atoms cannot be seen in the spectrum at all because of the fast tunneling reaction $\mathrm{H}_{2}+\mathrm{D} \rightarrow \mathrm{HD}+\mathrm{H}$. 


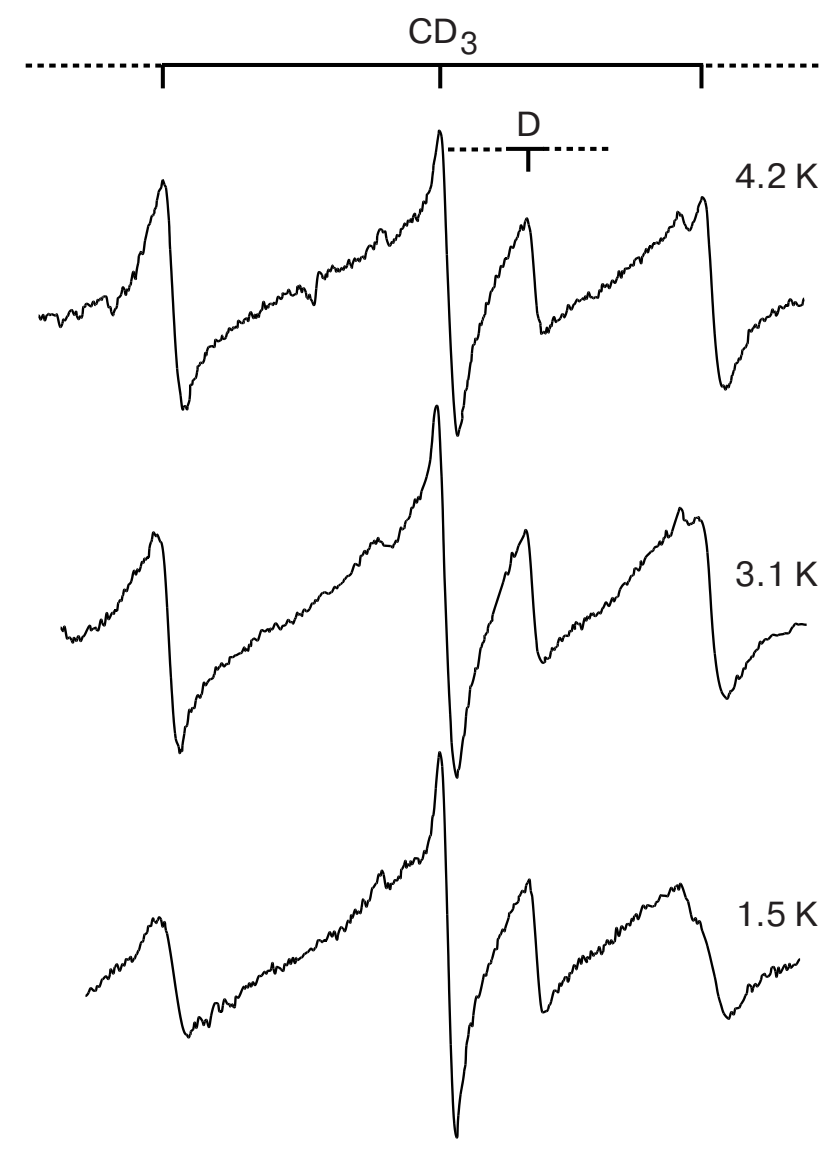

Fig. 8. Temperature-dependent central part of the $\mathrm{CD}_{3}$ radical matrix-isolated in solid Ne. The substrate temperature during deposition $T_{\text {sub }}=4.2 \mathrm{~K}$ at different sample temperature $T_{\text {samp }}$, K.

It is well known that the high-temperature $\mathrm{CHD}_{2}$ spectrum exhibits a «binomial» relative intensity ratio 1:2:3:2:1 for the quintet components. It has been found previously [5] that in the argon matrix the quintet transforms into a triplet with decreasing temperature from $10 \mathrm{~K}$ to $4.2 \mathrm{~K}$. In our experiments, the spectrum of $\mathrm{CHD}_{2}$ in $\mathrm{D}_{2}$ and $\mathrm{Ne}$ was still a double quintet at $4.2 \mathrm{~K}$. In $\mathrm{Ne}$ particularly, this spectrum showed, though, an intensity distribution different from the binomial one, which was evident from the fact that the ratio of the line amplitude at $m_{F}(D)=-1$ to the outermost one at $m_{F}(D)=-2$ was about 3 . This deviation from the binomial distribution is particularly evident in the $\mathrm{H}_{2}$ matrix. At sample temperatures as low as $1.5 \mathrm{~K}$ the transition at $m_{F}(D)=-2$ disappeared in both matrices, while the next two took similar amplitudes. Such a transformation of the shape of the spectrum suggests the transition from a mixture of the high- and low-temperature spectrum to the low-temperature double triplet. This change from high-temperature to the low-temperature spectrum for $\mathrm{CHD}_{2}$ corresponds very well to the case of the $\mathrm{CD}_{3}$ radical in $\mathrm{Ne}$ and $\mathrm{H}_{2}$ [11]. We could draw no conclusion about the $\mathrm{CHD}_{2}$ temper- ature behavior in solid $\mathrm{D}_{2}$ because of the poorly resolved spectrum. We have also failed to draw a conclusion about the appearance of the $\mathrm{CH}_{2} \mathrm{D}$ spectrum in both, $\mathrm{D}_{2}$ and Ne matrices, because the central $\mathrm{CH}_{2} \mathrm{D}$ triplet could not be seen against the strong $\mathrm{CD}_{3}$ transitions superimposed on it.

\section{Conclusion}

The present results verify the effect of temperature on the shape of the $\mathrm{CD}_{3}$ and $\mathrm{CHD}_{2}$ spectra first observed in $\mathrm{Ar}$ [5] with the samples obtained by x-ray radiolysis of a matrix containing $0.2 \mathrm{~mol} \% \mathrm{CH}_{4}$. Our data give also new information when comparing the temperature ranges of the spectrum transformation observed in Ref. 5 and here: $10-4.2 \mathrm{~K}$ and $4.2-1.5$, respectively. Since the spectrum changes we discuss are due mainly to changes in the populations of the lowest $J=0$ and $J=1$ states of trapped radicals, the difference in the range suggests that the energy interval between the above rotational states is significantly larger for radicals trapped in $\mathrm{Ar}$ [5] then in $\mathrm{H}_{2}, \mathrm{D}_{2}$, or Ne. In turn, a decrease in this interval for a trapped molecule in comparison with a free one is due to hindering of the rotation of the molecule in the matrix. Because the interaction energies for the $\mathrm{CD}_{3}-\mathrm{H}_{2}, \mathrm{CD}_{3}-\mathrm{D}_{2}$, and $\mathrm{CD}_{3}-\mathrm{Ne}$ pairs is lower than that of the $\mathrm{CD}_{3}-\mathrm{Ar}$ pair, one may expect a more free rotation of $\mathrm{CD}_{3}$ in $\mathrm{H}_{2}, \mathrm{D}_{2}$ and Ne, i.e., a smaller effect on the radical rotation than in Ar. Then, the present result suggests the phonon-rotation coupling for $\mathrm{CHD}_{2}$ and $\mathrm{CD}_{3}$ molecules in $\mathrm{H}_{2}, \mathrm{D}_{2}$, and Ne to be surprisingly higher than in Ar. On the other hand, the results for condensed samples are consistent with each other. Actually, a greater increase of the amplitude ratio with decreasing temperature for the $\mathrm{H}_{2}$ matrix as compared with that of $\mathrm{D}_{2}$, and Ne suggests that the $\mathrm{CD}_{3}-\mathrm{H}_{2}$ pair potential is lower than the other two: $\mathrm{CD}_{3}-\mathrm{D}_{2}$ and $\mathrm{CD}_{3}-\mathrm{Ne}$. One might suppose that the difference between our results and those of Ref. 5 originates from the greater impurity concentrations in the present study. However, there some reasons which make this unlikely. First, the major impurity (about $3 \mathrm{~mol} \%$ ) in the $\mathrm{H}_{2}$ matrix experiments was another hydrogen isotope, $\mathrm{D}_{2}$, which caused almost no shift of the lattice parameters and in the $\mathrm{CH}_{3}$-host molecule interaction. The concentration of $\mathrm{CH}_{4}$ was less than $0.1 \mathrm{~mol} \%$. Thus, the experiments with the molecular hydrogen matrix with a very small impurity concentration show a different temperature range for the EPR spectrum shape transformation as compared to [5]. Second, the narrow EPR lines in Ne matrices suggest rather regular surroundings. In our experiments, with $\mathrm{CH}_{4}$-doped $\mathrm{Ne}$ (about $0.1 \mathrm{~mol} \%$ ) we obtained lines with peak-to-peak widths of $0.12-0.15 \mathrm{G}$. The broadening observed in the present study (about 
$0.2-0.25 \mathrm{G})$ cannot be considered dramatic. Third, let the impurity concentration be $1-2 \mathrm{~mol} \%$. Then approximately $10 \%$ of the radicals have an admixture molecule in the nearest neighborhood, which could disturb the rotation motion of the radicals. The other $90 \%$ of radicals are trapped in the fine-grain crystallites. Thus, the first group exhibits hindered rotation, while the larger second group undergoes free rotation [5]. Even though, we would not see near binomial distribution at $4.2 \mathrm{~K}$, based on the temperature region found out in ref [5]. Indeed, the amplitude of the low-temperature singlet is so large that the above free rotating radicals even at sufficiently less concentration than suggested above would transform the spectrum shape into the low-temperature one.

Another conclusion which could be drawn is that the difference between our results and those of Ref. 5 lies in the difference of the sample preparation techniques: radiolysis in Ref. 5 and condensation from a gas discharge in the present study. This may lead to different matrix surroundings in these experiments, that is a larger matrix cage for the $\mathrm{CH}_{3}$ obtained by radiolysis. With this technique, the great amount of energy being absorbed locally may lead to a significant local lattice distortion. It was pointed out in Ref. 5 that the EPR line shape of $\mathrm{CH}_{3}$ below $40 \mathrm{~K}$ was reversibly changed with temperature. It is well known that annealing of Ar matrix at $30 \mathrm{~K}$ give rise to relaxation of structural defects. Althoudh, the same supposedly holds for $\mathrm{CD}_{3}$, it is not stressed in the text that deuterated methyl radicals also showed reversible changes in the same large temperature range of 4.2 through $40 \mathrm{~K}$.

It cannot be ruled out completely that the difference in the matrix preparation techniques would contribute to the difference in the temperature ranges. On the other hand, this contribution is unlikely a deciding factor, because both techniques utilize condensation of a gaseous sample onto a cold surface.

We suggest that the key to this question of different temperature ranges for the spectrum shape transformation lies with the integral intensity of the spectra. If we take this into account, then the results in Ref. 5 and the present data become consistent. Indeed, it is pointed out in Ref. 5 that at the temperature of $4.1 \mathrm{~K}$ the linewidth of the central singlet is $0.06 \mathrm{G}$, whereas the high-temperature transitions are about 4 times broader than the central singlet. Given the relative amplitude of the singlet to the outermost transitions of 105:1 [5] and that the integral intensity is proportional to the product of the amplitude and the square of the linewidth, we estimate the integral intensity ratio of the singlet to the high-temperature septet as 1:4. Since the relative population of rota- tional levels is determined by ratio of integral intensities of lines corresponding to different states, the above result suggests that the majority of the $\mathrm{CH}_{3}$ radicals are at higher rotational levels. Now, let the EPR lines be of the same width, i.e., broadened due to certain mechanisms: superhyperfine interaction in the $\mathrm{H}_{2}$ and $\mathrm{D}_{2}$ matrices and, possibly, structural defects in Ne. Then a relative amplitude ratio of the singlet to its nearest neighbor transition would be about 13:6 at $4.2 \mathrm{~K}$, which is comparable with our results: $12: 6.5$ in $\mathrm{H}_{2}$ [11], 8:6 in $\mathrm{D}_{2}$, and 7.5:5.4 in Ne.

One more conclusion which stems from the above integral intensity ratio of 1:4 concerns the temperature range for the spectrum shape transition. It is obvious that the population of the lowest rotational level is far from saturation at $4.1 \mathrm{~K}$, and it should keep rising with further decrease temperature. Given lines of equal width, the most prominent spectrum shape transformation would be expected at temperatures below $4.1 \mathrm{~K}$, which is the case in the present study. Because of the unchanged spectrum linewidth with changing temperature, the temperature dependence of the amplitudes in Fig. 5 reflects the relative population of different states. It follows from Fig. 5 that there is a difference between matrices in population of levels at a given temperature. Thus, the results suggest a hindered rotation. This follows from both: the low temperature range for the spectrum transformation as compared to the energy gap between the two lowest rotational levels of the free $\mathrm{CH}_{3}$ radical , and the distinction in the temperature dependence of the level populations for different matrices.

It is of interest now to study an effect of matrix deposition conditions as well as impurity concentration on the rotational barrier. The quench-condensed films of $\mathrm{Ar}$ and $\mathrm{Kr}$ are known to contain an appreciable number of structural defects. For pure Kr quench-condensed onto a $5 \mathrm{~K}$ substrate, the average number of planes between stacking faults is estimated as equal to $11 \pm 3$, which is a very high density [13]. The matrices studied in the present work have considerably lower annealing temperatures then $\mathrm{Kr}$ and $\mathrm{Ar}$, and would contain a much lower density of structural defects. As for impurities, it is not a direct effect on the rotation of the molecule that is interesting, because it is hard to observe (see above), but an indirect effect through the influence on the matrix morphology [13]. In turn, this influence is more prominent for mixtures of two substances differing strongly in molecular parameters [13], like $\mathrm{Kr}: \mathrm{D}_{2}$ and $\mathrm{Ar}: \mathrm{D}_{2}$, but not $\mathrm{Ne}: \mathrm{D}_{2}$.

1. R.L. Morehouse, J.J. Christiansen, and W. Gordy, J. Chem. Phys. 45, 1751 (1966).

2. G.S. Jackel and W. Gordy, Phys. Rev. 176, 443 (1968). 
3. C.K. Jen, S.N. Foner, E.L. Cochran, and V.A. Bowers, Phys. Rev. 112, 1169 (1958).

4. E.Ya. Misochko, V.A. Bendersii, A.U. Goldschleger, A.V. Akimov, A.V. Benderskii, and C.A. Wight, J. Chem. Phys. 105, 3146 (1997).

5. T. Yamada, K. Komaguchi, M. Shiotani, N.P. Benetis, and A.R. Sornes, J. Chem. Phys. A103, 4823 (1999).

6. Yu.A. Dmitriev and R.A. Zhitnikov, J. Chem. Phys. 122, 163 (2001).

7. H.M. McConnel, J. Chem. Phys. 29, 1422 (1958).

8. J.H. Freed, J. Chem. Phys. 43, 1710 (1965).
9. A.M. Bass and H.P. Broida (eds.), Formation and Trapping of Free Radicals, Academic Press, New York and London (1960).

10. K. Toriyama, M. Iwasaki, and K. Nunome, J. Chem. Phys. 71, 1698 (1979).

11. Yu.A. Dmitriev and R.A. Zhitnikov, Fiz. Nizk. Temp. 29, 595 (2003) [Low Temp. Phys. 29, 519 (2003)].

12. R.A. Zhitnikov and Yu.A. Dmitriev, Astronomy and Astrophysics 386, 129 (2002).

13. M.A. Strzhemechny, N.N. Galtsov, and A.I. Prokhvatilov, Fiz. Nizk. Temp. 29, 699 (2003) [Low Temp. Phys. 29, 522 (2003)]. 